\title{
RSSP: RSVP SESSION SET-UP PROTOCOL FOR RSVP-INCAPABLE MULTIMEDIA APPLICATIONS
}

\author{
Xiaobao Chen \\ Lucent Technologies \\ xchen1@lucent.com
}

\author{
Andrea Paparella \\ Lucent Technologies \\ apaparella@lucent.com
}

\author{
Ioannis Kriaras \\ Lucent Technologies \\ ikriaras@lucent.com
}

\begin{abstract}
RSVP is the QoS signalling protocol used with Integrated Service Framework for QoS provisioning in IP networks. The existing RSVP mechanism requires that an RSVP daemon be embedded with the applications so as to initiate RSVP sessions. This requirement has been envisaged to be a major limitation over the use of RSVP and the associated QoS control mechanisms for those applications or platforms which are either RSVP-incapable or impossible to be modified to introduce a built-in RSVP capability. This paper introduces a mechanism, RSVP Session Set-up Protocol, which overcomes the limitations of existing RSVP implementations by initiating RSVP sessions for RSVP-incapable applications running on different platforms. The proposed mechanism features application and platform independence and eliminates the need for built-in RSVP processing capability for those applications or terminals of short battery life and limited processing power. Moreover it facilitates dynamic QoS provision for wireless mobile devices during hand-off control.
\end{abstract}

Keywords: QoS, RSVP, Proxy Server, and Multimedia

\section{INTRODUCTION}

QoS provisioning and Differentiating Classes of Services have been a major issue in research, commercial, industrial, and standardisation activities in both wired and wireless telecommunications. Two major elements need to be provided for a successful provisioning of QoS control: the QoS signalling mechanism and the traffic control. As one of the many alternatives in providing QoS, IETF's Integrated Services architecture 
provides a framework for applications to choose between multiple controlled levels of delivery services for their traffic flows. Applications need to communicate their QoS requirements to the QoS-control capable nodes along the transit path, as well as for the network nodes to communicate between one another. RSVP, a receiver-oriented QoS signalling protocol, is proposed by IETF as an approach to provide QoS requests to all routers along the transit path of the traffic flows and to maintain the state necessary in the router required to provide different levels of delivery services for applications using RSVP to signal their QoS requests. All existing RSVP implementations have been platform dependent and require an RSVP functional module or a daemon to be embedded with applications so as to initiate a RSVP session. This has incurred serious limitations of using RSVP and IntServ for QoS provisioning for those applications and platforms that are RSVP-incapable or difficult or even impossible to be changed to build the RSVP functionality. Moreover it makes it difficult to support seamless hand-off with QoS control using RSVP for a mobile device or terminal which roams between different base stations and thus requires instant updating of an RSVP session between itself and its communication peer(s). It is, therefore, an major motivation for inventing a mechanism as proposed in this paper to overcome the limitations of existing RSVP implementation mechanisms and facilitate the use of RSVP in a wireless mobile environment. Section 2 introduces the proposed mechanism, RSVP Session Set-up Protocol (RSSP) and RSSP Agent and Client, with detailed discussions on its design and implementation. Section 3 provides a performance evaluation. In Section 4 a review of the related work is given. Finally Section 5 concludes the paper with some observations.

\section{RSVP SESSION SET-UP PROTOCOL (RSSP) AND ITS DESIGN}

RSSP is defined to specify the RSVP session invocation procedures between a RSSP agent and a RSSP client. A RSSP agent is set up which is able to process standard RSVP messages and, at the same time, is able to intercept the RSVP messages destined for RSSP clients that have registered with the RSSP agent.

\subsection{RSSP Agent Discovery}




\section{(i) RSSP Agent Advertisement}

The location of an RSSP agent is advertised by sending (multicasting or broadcasting) Client Request Messages (CRQM). The CRQM message can be an UDP packet that bears the information about the current location and the provided services by the RSSP agent. A RSSP client uses the information in these advertisements to register and issues an RSSP service request to a RSSP agent. The advertisement information as carried in a CRQM can include:

- The current location, specifically the IP address, of the RSSP Agent.

- The service access point, specifically a port number, on which the RSSP Agent is listening for any incoming service request and to which a RSSP client sends its RSSP service request.

- The lifetime of the agent. The default lifetime is eternal.

- The security control information such as security key or index used between the proxy agent and the client.

- The services provided by the RSSP agent. For example, it may only provide a subset of IntServ services in its RSVP session set-up service (Controlled Load or Guaranteed Service only). It may provide all or just one or two of the three reservation styles, FF, WF, SE.

\section{(ii) RSSP Agent Soliciting}

For a RSSP client which requires the set-up of RSVP session but does not have an agent registry in its own list of valid RSSP agents, it sends (multicasting or broadcasting) messages called Agent Soliciting Message (ASM). An RSSP agent in the vicinity running in its rormal service state will respond with a unicast agent advertisement message Agent Response Message (ARM) sent directly to the soliciting client. After receiving the ARM, the soliciting client responds by sending a Client Registration Message (CRGM) to the agent as it does upon receiving an Agent Advertisement Message. The ASM can be an UDP message with a broadcast address as its destination address. In addition, it contains:

- The location, specifically the IP address, or other identity information of the client.

- The requested RSVP session services, including the reservation style, unicast or multicast service, Controlled-Load or Guaranteed service, etc. 
- The requested lifetime of service. The lifetime has to be specifically indicated otherwise the RSSP agent rejects the RSVP service request.

- The security control information such as the encryption key.

The ARM message can be an UDP message with the soliciting client's address as the destination address. It contains:

- The confirmation of the validity of the solicitation from the client.

- The availability or the confirmation of the requested RSVP session service.

- The suggested lifetime of the service. It should be no longer than the required service time.

- The security control information such as the encryption key or the authentication information to be used during the interaction between the client and the agent.

A RSSP agent advertisement message, CRQM, uses a broadcast UDP message to advertise its presence, location and services.

\subsection{RSSP Agent Registration}

A RSSP client registers with its RSSP agent by sending a unicast Client Registration Message (CRGM) message to the Agent. The CRGM message can be an UDP message sent to the RSSP agent with specific QoS/RSVP session service requirements including:

- The explicit QoS requirements such as the specifications on average data rate, maximum delay, delay variation, peak rate and packet loss rate.

Or

- The implicit QoS requirement such as specific coding algorithm $\mathrm{s}$ and the codecs being used by the clients, e.g. H.263, MPEG-4, etc.

- The selected signalling protocol type, Type " 1 " is reserved for RSVP.

The selected QoS control type, Type "1" is reserved for Integrated Service.

- The requested service time during which the client expects the RSSP agent to set up and maintain its RSVP session(s).

- Security control information such as the authentication information and encryption key. 
An CRGM message can be one of the following five types:

- RSSP_REQ: A RSSP service request message sent from a client to a RSSP agent. It is usually sent by a client serving as a data sender to invoke the transmission of RSVP Path messages through the RSSP agent.

- RSSP_IND: An RSSP service indication message issued by a RSSP agent and sent to a specific RSSP client serving as a data receiver. It indicates the arrival of Path messages at the RSSP agent for a particular flow to be received by the client.

- RSSP_REP: An RSSP session service response message issued by a RSSP client as an indication to the RSSP agent to start sending RSVP Resv messages.

- RSSP_CON: An RSSP session service confirmation message issued by a RSSP agent at the data sender's side to confirm the arrival of Resv messages at the RSSP agent and the successful set-up of a RSVP session.

- RSSP_REJ: An RSSP session service rejection message issued by a RSSP agent as an indication of the failure of setting up of a RSVP session, in particular, when the RSSP agent receives PathErr/ResvErr messages reporting errors during the set-up of Path/Resv states at certain routers.

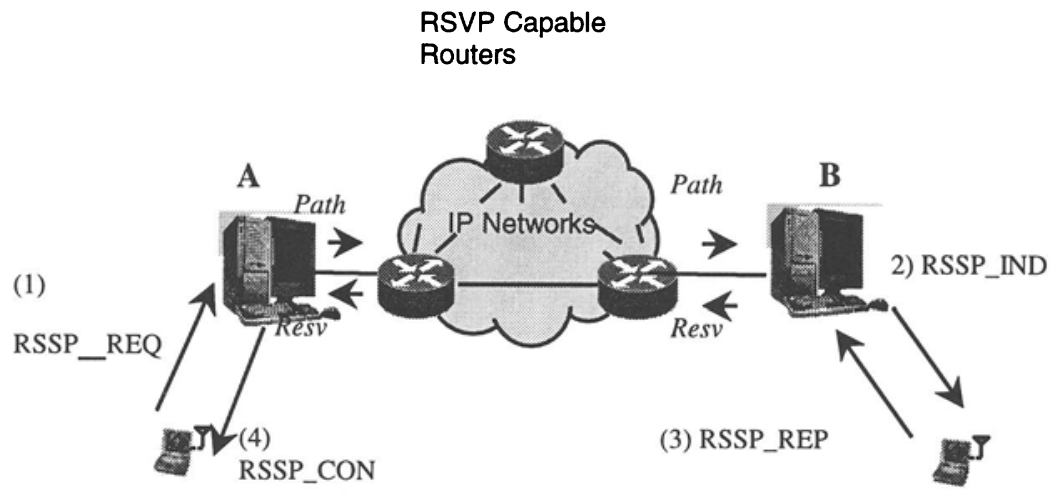

Figure 1. The RSSP Agent Registration Process

Figure 1 shows the control procedures and the control messages exchanged between a RSSP client and its RSSP agent and between the peer RSSP 
agents. It demonstrates an example for using RSVP as the QoS signalling protocol to inform the intermediate IP routers of the QoS specifications for a specific data transmission and set up and maintain the RSVP states (Path/Resv states). RSSP agent A serves a RSSP client to be a data sender while RSSP gent B serves a RSSP client to be a data receiver. A RSVP session is maintained by the RSSP agent(s) which sends Path/Resv message periodically to refresh the RSVP soft-states until an explicit agent deregistration request is received or the requested service time expires.

\section{RSSP SERVERS AND PERFORMANCE EVALUATION}

To test the control effectiveness and evaluate the feasibility of the proposed mechanism, two RSSP Servers are set up with the functions of RSSP agent's and connected to our multimedia QoS test-bed. The network configuration of the test-bed is shown in Figure 2. Two sub-networks including both wired and wireless multimedia servers and terminals are interconnected through RSVP-capable routers. Multimedia servers provide video/audio/web services to the multimedia access and display terminals as what is shown as the RSSP client. RSSP clients (the wireless terminal and the multimedia server) register with its local RSSP Server to invoke the setup of a RSVP session across the inter-networking routers. A successful RSSP Server operation in response to a RSVP session set-up request from the multimedia server and the terminal results in the establishment of a RSVP session along the transit path of the multimedia flow(s) between the multimedia server and the multimedia terminal(s).

\subsection{Qualitative Performance Evaluation}

For the current configuration of the test-bed, Video-on-Demand (VoD) Servers and the multimedia terminals run on machines running Windows 95/NT4.0/SunSoloaris where no changes or modifications are introduced to make the VoD servers and our wired/wireless terminals RSVP-capable. To test the control effectiveness of the proposed RSSP scheme, two multimedia display terminals (laptops) running Windows 95 are used to request the same video service from the VoD server. The VoD session and the RSVP session configuration parameters are shown in Table 1 and Table 2, respectively. Two scenarios with different network loading conditions are investigated, one without network overload and the other with network overloaded and thus with traffic congestion at the routers. 


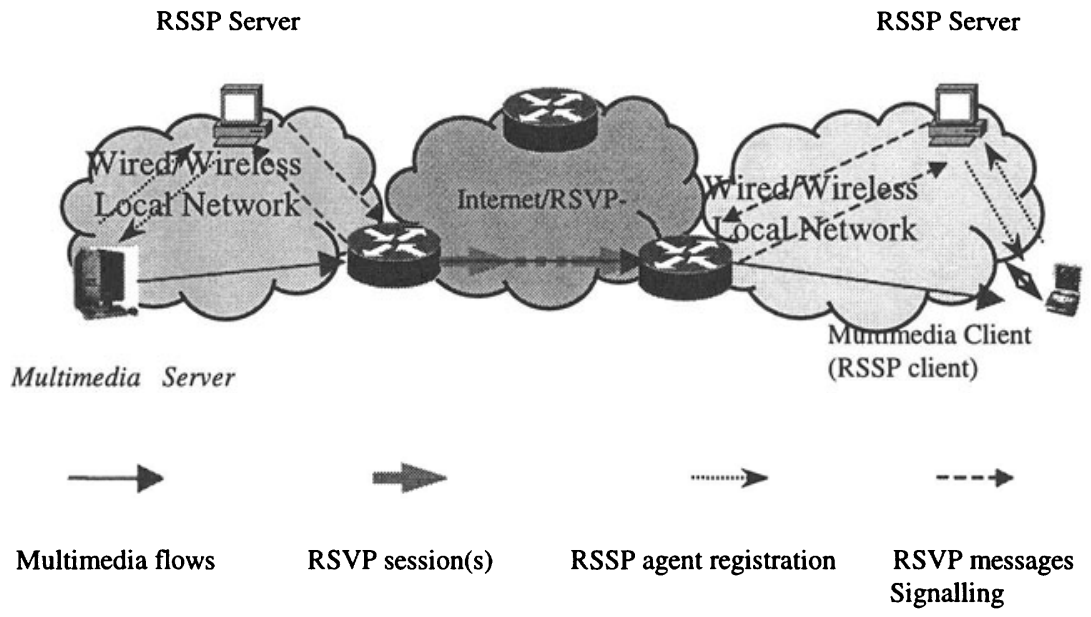

Figure 2. RSSP/QoS Multimedia Test-bed Network Configuration

For the non-overloading scenario, the routers are configured to provide a total transmission bandwidth of no less than $2 \mathrm{Mbps}$. It is observed that the perceptive video and audio display quality at both terminals are satisfactory without observable delay or standstill or broken images. There is no perceivable loss of audio and video inter-media synchronisation.

\begin{tabular}{|l|l|l|l|l|c|}
\hline $\begin{array}{l}\text { Multimedia } \\
\text { Terminals }\end{array}$ & $\begin{array}{l}\text { Source/Port } \\
\text { Number }\end{array}$ & $\begin{array}{l}\text { Dest./ } \\
\text { Port } \\
\text { Number }\end{array}$ & Protocol & Codec & $\begin{array}{c}\text { Frame Rate } \\
\text { (Frames/sec) }\end{array}$ \\
\hline Terminal I & 135.86 .224 .86 & $\begin{array}{l}192.128 .71 . \\
20 /\end{array}$ & UDP & H.261 & 33 \\
& $/ 1555$ & 1122 & & & \\
\hline Terminal II & 135.86 .224 .86 & $\begin{array}{l}192.128 .71 . \\
68 /\end{array}$ & UDP & H.261 & 33 \\
& 11556 & 1234 & & & \\
\hline
\end{tabular}

Table 1. VoD Session Parameters

For the network-overloading scenario the routers are configured to provide a total transmission bandwidth of $1 \mathrm{Mbps}$. It is clearly observed that the audio/video perceptive quality for terminal $I$ is guaranteed in that no difference is observable in the perceptive qualities from that in the nonoverloading network scenario. However, for terminal II without RSVP session service and thus no QoS control over its VoD session, a serious deterioration in perceptive audio/video quality is observed. A proximately two seconds delay is observed in the video display in comparison with that 


\begin{tabular}{|l|l|l|l|l|l|l|l|l|}
\hline $\begin{array}{l}\text { RSVP } \\
\text { Session }\end{array}$ & $\begin{array}{l}\text { Source/ } \\
\text { Port No. }\end{array}$ & $\begin{array}{l}\text { Dest./ } \\
\text { Port No. }\end{array}$ & $\begin{array}{l}\text { Pro- } \\
\text { tocol }\end{array}$ & Service & $\begin{array}{l}\text { Avg./ } \\
\text { Peak } \\
\text { Rates } \\
\text { (kbps) }\end{array}$ & $\begin{array}{l}\text { Min/Max } \\
\text { MTU } \\
\text { (bytes) }\end{array}$ & $\begin{array}{l}\text { Buf. } \\
\text { (bytes) }\end{array}$ & $\begin{array}{l}\text { Slack } \\
\text { Term } \\
\text { (us) }\end{array}$ \\
\hline Session I & $\begin{array}{l}135.86 .2 \\
24.86 / 15 \\
55\end{array}$ & $\begin{array}{l}192.128 . \\
71.20 / 11 \\
22\end{array}$ & UDP & $\begin{array}{l}\text { Guarante } \\
\text { ed }\end{array}$ & $\begin{array}{l}650 / \\
750\end{array}$ & $64 / 9138$ & 81250 & 100 \\
\hline $\begin{array}{l}\text { Session } \\
\text { II }\end{array}$ & $\mathrm{X}$ & $\mathrm{X}$ & $\mathrm{X}$ & $\mathrm{X}$ & $\mathrm{X}$ & $\mathrm{X}$ & $\mathrm{X}$ & $\mathrm{X}$ \\
\hline
\end{tabular}

Table 2. RSVP Sessions for Terminal I and II.

for terminal I. Broken and even standstill pictures happen from time to time during its traffic bursts. A total loss of audio and video inter-media synchronisation is also clearly observed.

\subsection{Quantitative Performance Evaluation}

In order to investigate detailed behaviours of QoS control of the RSVPcapable routers under network overloading conditions with RSSP Servers, further experiments are carried out with detailed statistics analysis on a series of packet transmission quality parameters, including effective received data rates, packet loss, maximum end-to-end transmission delay and delay variations (jitter). Traffic generators and receivers are set up running on separate machines in the two sub-networks as shown in Figure 2. At the transmission side, the traffic generator is configured to transmit five simultaneous traffic streams with transmission bursts. The detailed configuration parameters are shown in Table 3.

A RSVP session is set up between the traffic generator and the traffic receiver for Stream II via the RSSP Server in the local sub-network across the inter-networking routers. The RSVP Session State for Stream II is shown in Table 4.

At the receiver side the traffic is monitored and receiving statistics of the all five streams are collected. In order to test the control effectiveness of the RSVP session established through the RSSP Server under the network overloading condition, the routers are configured to provide maximum $500 \mathrm{kpbs}$ bandwidth in total so that the routers work under over-loaded conditions with five simultaneous streams, each of which generates an average date rate of $128 \mathrm{kbps}$. 


\begin{tabular}{|c|c|c|c|c|c|c|c|c|}
\hline $\begin{array}{l}\text { Traffic } \\
\text { Streams }\end{array}$ & $\begin{array}{l}\text { Src./ } \\
\text { Port } \\
\text { Number }\end{array}$ & $\begin{array}{l}\text { Dest/. } \\
\text { Port } \\
\text { Number }\end{array}$ & $\begin{array}{l}\text { Traffic } \\
\text { Pattern }\end{array}$ & Protocol & $\begin{array}{l}\text { Avg. } \\
\text { Data } \\
\text { Rate } \\
\text { (kbps) }\end{array}$ & $\begin{array}{l}\text { Peak } \\
\text { Data } \\
\text { Rate } \\
\text { (kbp } \\
\text { s) } \\
\end{array}$ & $\begin{array}{l}\text { Packe } \\
\mathbf{t} \\
\text { Size } \\
\text { (bytes } \\
\end{array}$ & $\begin{array}{l}\text { Tx. Time } \\
\text { (packets) }\end{array}$ \\
\hline Stream I & $\begin{array}{l}135.86 .22 \\
4.50 / 4000\end{array}$ & $\begin{array}{l}192.128 . \\
71.20 / 50 \\
01\end{array}$ & Poisson & UDP & 128 & 256 & 1000 & 100000 \\
\hline $\begin{array}{l}\text { Stream } \\
\text { II }\end{array}$ & $\begin{array}{l}135.86 .22 \\
4.50 / 4000\end{array}$ & $\begin{array}{l}192.128 . \\
71.20 / 50 \\
02\end{array}$ & Poisson & UDP & 128 & 256 & 1000 & 100000 \\
\hline $\begin{array}{l}\text { Stream } \\
\text { III }\end{array}$ & $\begin{array}{l}135.86 .22 \\
4.50 / 4000\end{array}$ & $\begin{array}{l}192.128 . \\
71.20 / 50 \\
03\end{array}$ & Poisson & UDP & 128 & 256 & 1000 & 100000 \\
\hline $\begin{array}{l}\text { Stream } \\
\text { IV }\end{array}$ & $\begin{array}{l}135.86 .22 \\
4.50 / 4000\end{array}$ & $\begin{array}{l}192.128 . \\
71.20 / 50 \\
04\end{array}$ & Poisson & UDP & 128 & 256 & 1000 & 100000 \\
\hline $\begin{array}{l}\text { Stream } \\
\mathrm{V}\end{array}$ & $\begin{array}{l}135.86 .22 \\
4.50 / 4000\end{array}$ & $\begin{array}{l}192.128 \\
71.20 / 50 \\
05\end{array}$ & Poisson & UDP & 128 & 256 & 1000 & 100000 \\
\hline
\end{tabular}

Table 3. Traffic Generation Statistics

\begin{tabular}{|c|c|c|c|c|c|c|c|c|}
\hline $\begin{array}{l}\text { RSVP } \\
\text { Session }\end{array}$ & $\begin{array}{l}\text { Src./ } \\
\text { Port } \\
\text { Num } \\
\text { ber }\end{array}$ & $\begin{array}{l}\text { Dest. / } \\
\text { Port } \\
\text { Number }\end{array}$ & Protocol & Service & $\begin{array}{l}\text { Avg./ } \\
\text { Peak } \\
\text { Rates } \\
\text { (kbps) }\end{array}$ & $\begin{array}{l}\text { Min/Max } \\
\text { MTU } \\
\text { (bytes) }\end{array}$ & $\begin{array}{l}\text { Buf. } \\
\text { (bytes } \\
\text { ) }\end{array}$ & $\begin{array}{l}\text { Slack } \\
\text { Term } \\
\text { (us) }\end{array}$ \\
\hline $\begin{array}{l}\text { Stream } \\
\text { II }\end{array}$ & $\begin{array}{l}135 \\
86.2 \\
24.5 \\
0 / 40 \\
00\end{array}$ & $\begin{array}{l}192.128 \\
71.20 / 50 \\
02\end{array}$ & UDP & $\begin{array}{l}\text { Guaran } \\
\text { teed }\end{array}$ & $128 / 230$ & $64 / 9138$ & 16000 & 100 \\
\hline
\end{tabular}

Table 4. RSVP Session Configuration for Stream II.

Two separate experiments are carried out. In the first experiment, no RSVP session is set up for any of the streams and therefore the routers provide best-effort service to all streams. In the second experiment, a RSVP session is set up for Stream II as shown in Table 4. The measured performance statistics include the effective received data rate, packet drop, end-to-end maximum transmission delay and the delay variation. The results are shown in Figure 3, 4, 5 and 6, respectively.

Figure 3 shows that, for stream II with a RSVP-enabled QoS provision, it receives data at the average rate of $129.8 \mathrm{kbps}$. The minor difference from 
its requested average data rate $128 \mathrm{kbps}$ is caused by the uneven arrival of packet transmission which uses Poisson distribution and thus the data rate varies in time. The measured received data rate is calculated on the average over 100000 packets. Therefore, there is a slight variation from the requested average transmission bandwidth. It is also observed that all other streams, III, IV, V and I, receive data at an average rate of equable share of the left bandwidth after the resource reservation for stream II. It is also interesting to point out that all five streams will share the total available bandwidth when no RSVP service is invoked as shown on the right side of

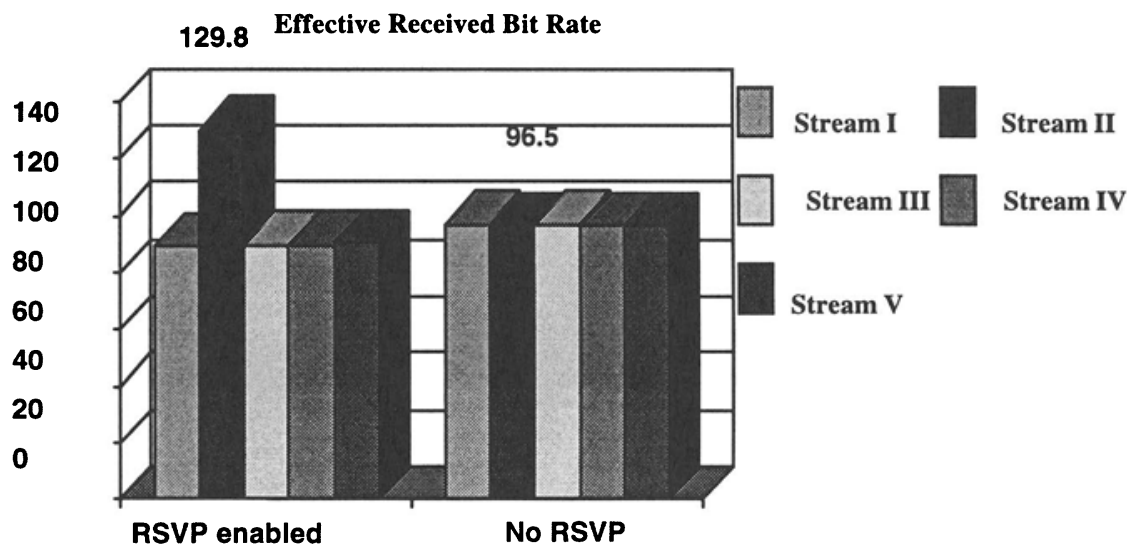

Figure 3. Per-Flow Effective Bandwidth with/without RSVP Sessions

Figure 3. This has clearly signified the best-effort service nature of IP routers when there is no resource reservations. The distribution of packet loss is shown in Figure 4. It clearly shows that stream II is subject to no packet loss while all other streams suffer from dramatic high level of packet loss due to the traffic congestion at each router and uncontrolled packet drop. It is also observed that the overall network over-load does not affect the packet loss level of stream II with RSVP-enabled resource reservation as long as the reserved resources (buffer length) can accommodate the burst of its traffic, while for all other traffic streams without resource reservations, more network overload leads to higher levels of packet loss. Again for the best-effort service nature of IP networks as featured by each IP router when RSVP or other QoS provisioning mechanism is not in operation, all streams 
will be subject to the same level of packet drop as shown by the histogram on the right-hand side in Figure 4

It can be seen from Figure 5 that stream II with RSVP enabled QoS control over its data packets at each router has its end-to-end delay minimised in comparison to all other streams without RSVP session service. Similar to the bandwidth and packet loss control as shown in Figure 3 and 4, the same level of maximum end-to-end transmission delays is incurred on all streams as the best-effort service provided by each router if there is no QoS control enabled. It needs to be noted that the values as shown in Figure 5 are measured by synchronising the system clocks across the networks, i.e.,

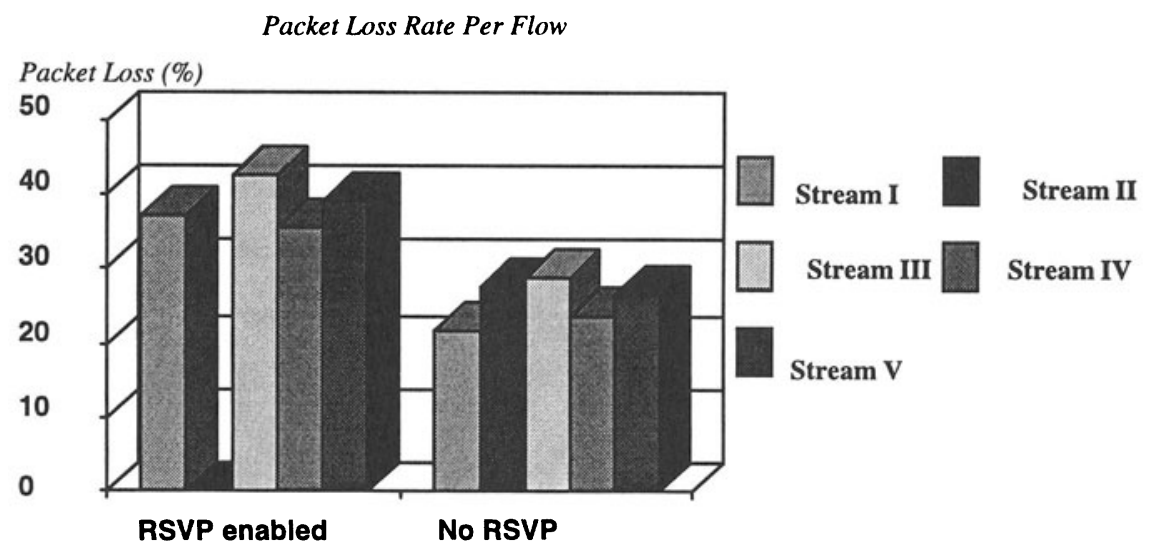

Figure 4. Per-Flow Packet Loss with/without RSVP Sessions

between the traffic generators and receivers as well as across the routers, via NTP running as a daemon in each machine. The estimated clock synchronisation skew achieved by the NTP is about half a second under the light-load network condition. Although the Integrated Service QoS Framework is deployed together with RSVP for QoS provisioning in the current test-bed, according to the IntServ RFC specifications, it does not provide control over the maximum achievable delay variations. But the actual delay variation as experienced by each packet for stream II is limited and dramatically reduced in comparison with those experienced by all other streams without RSVP enabled resource reservations. This is clearly shown by the histogram on the left-hand side in Figure 6. 


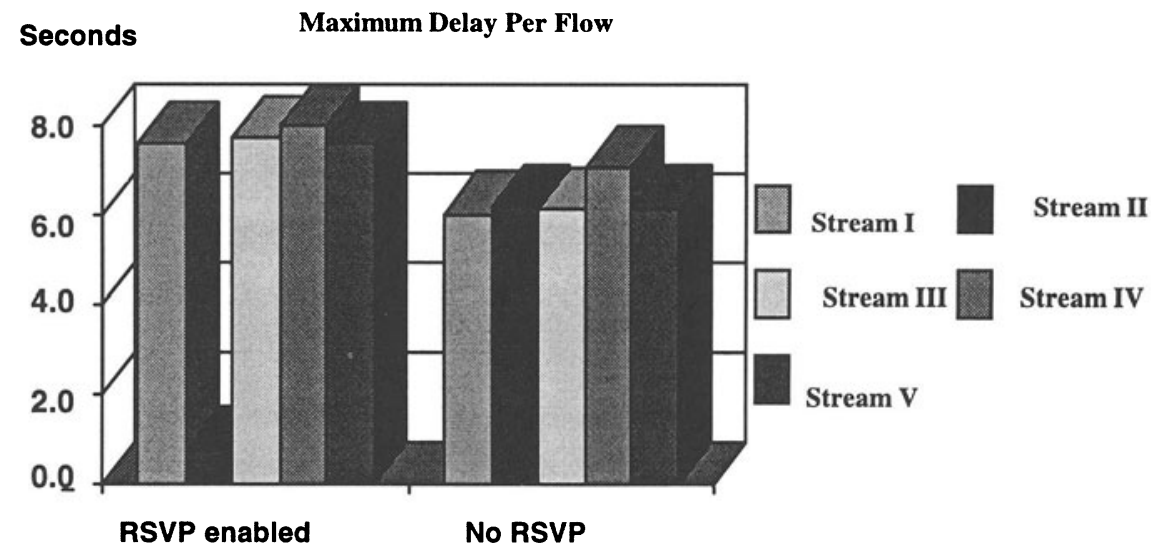

Figure 5. Per-Flow Maximum End-to-End Transmission Delay with/without RSVP Sessions

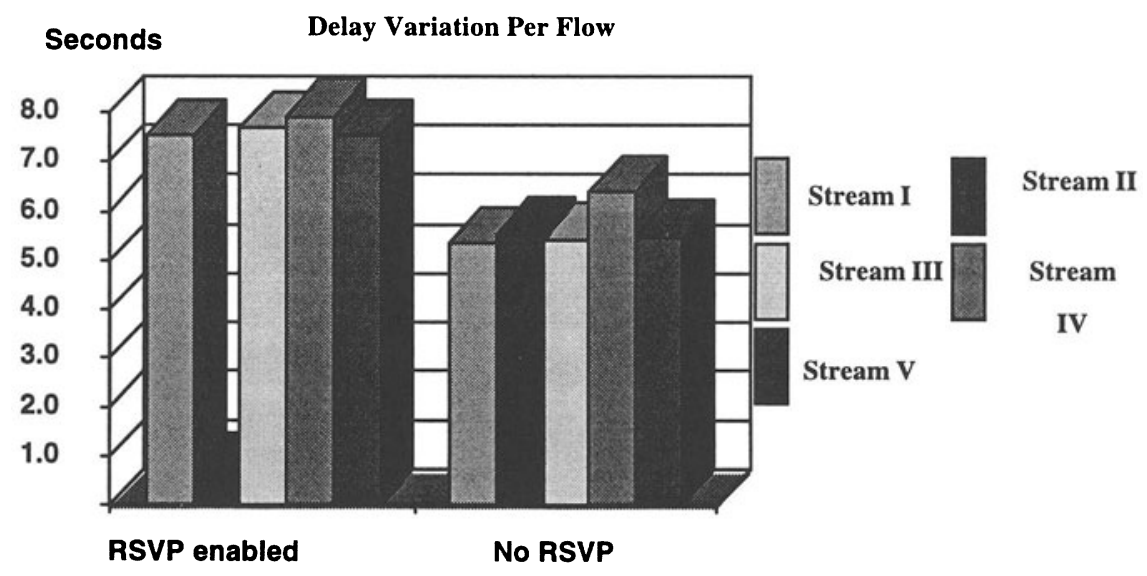

Figure 6. Per-Flow Delay Variation with/without RSVP Sessions

Similar to the measurement of end-to-end delay, the measured delay variation is subject to the clock synchronisation skew which fluctuates over time, in particular, during the network over-load condition which will cause 
long delay and loss of NTP messages used to refresh the system clocks across the networks.

\section{RELATED WORK}

3Com supports RSVP both on the router family NetBuilderII and on the switch family CoreBuilder. What is particularly interesting is the CoreBuilder 3500. It is a 10/100 Mbps Ethernet L2/L3 switch that provides differentiated levels of QoS for multimedia applications. As an alternative approach to the SBM mechanism, using RSVP for local resource reservations in CoreBuilder is obviously a complement to the proposed RSSP Server that provides resource reservations over inter-networking nodes. Ascend Communications (http://www.ascend.com) GRF Multigigabit Router supports both Integrated Service/RSVP, offering Controlled Load and Guaranteed services, and Differentiated Service models. At the time of writing the paper, it is still in development status. The Cisco Systems routers provide differentiated levels of QoS for multi-media applications. They support Integrated Service/RSVP Control Load service, which are RSVP and IntServ compliant. The proposed RSSP and the implementation of RSSP Server/Client has been tested with the Cisco Systems Routers for RSVP/IntServ QoS control over multimedia traffic with full inter-operability. There have been other RSVP implementations on MS-Windows (95/NT4.0/98/NT5.0), Sun Solaris, DEC Alpha machines and Line. From their descriptions they are not targeting at resolving the application and platform dependence problems of RSVP.

\section{CONCLUSION AND FUTURE WORK}

RSVP Session Set-up Protocol, RSSP, is proposed in this paper to overcome the limitations of current RSVP design and implementation such as application and system independence which can cause serious limitations to the deployment of RSVP services over simple machines and terminals with limited and expensive processing powers to build a full RSVP session setup and maintenance functions. It also provides those applications that are difficult or even impossible to embed an RSVP daemon or its equivalent functions within the platforms. Both qualitative and quantitative tests have been carried out and the experimental results demonstrate the control effectiveness through the RSSP Server in QoS provisioning over real-time applications. The proposed scheme has been successfully deployed in providing QoS control over multimedia traffic to and between mobile nodes 
in our mobile multimedia QoS control test-bed. Through the design and implementation work the proposed mechanism has been proved to play a significant role in providing dynamic QoS provisioning during the seamless hand-off control of mobile multimedia terminals.

\section{References}

[1] S. Shenker, J. Wroclawski, RFC 2215 "General Characterisation Parameters for Integrated Service Network Elements", RFC 2215, September 1997

[2] S. Shenker, J. Wroclawski, "Network Element Service Specification Template", RFC 2216, September 1997.

[3] J. Wroclawski, "The Use of RSVP with IETF Integrated Services", RFC2210, September 1997.

[4] J. Wroclawski, "Specification of the Controlled-Load Network Element Service", RFC2211, September 1997.

[5] S. Shenker, C. Partridge, R. Guerin, "Specification of Guaranteed Quality of Service", RFC2212, September 1997.

[6] R. Braden, D. Clark, S. Shenker, "Integrated Services in the Internet Architecture: an Overview", Informational RFC1633R, June, 1994.

[7] R. Braden, L. Zhang, S. Berson, S. Herzog, S. Jamin, "Resource ReSerVation Protocol (RSVP) - Version 1 Functional Specifiction", September 1997.

[8] A. Mankin, F. Baker, B. Braken, S. Bradner, M. O'Dell, A. Ramanow, A. Weinrib, L. Zhang, "Resource ReServation Protocol (RSVP) Version 1 Applicability Statement", Informational RFC2208, September 1997.

[9] R. Braden, L. Zhang, "Resource ReSerVation Protocol (RSVP) -Version 1 Message Processing Rules", RFC2209, September 1997.

[10] R. Yavatkar, D. Hoffman, Y. Bernet, F. Baker, M. Speer, "SBM (Subnet Bandwidth Manager): Protocol for RSVP-based Admission Control over IEEE 802-style Networks", IETF Internet-Draft <draftietf-issll-is802-sbm-06.txt>, March 1998.

[11] http://www.isi.edu/rsvp/DOCUMENTS/ietf_rsvp-qos_survey_02.txt 